\title{
Correlation of red cell distribution width with inflammatory markers and its prognostic value in patients with diabetes and coronary artery disease
}

\section{ABSTRACT}

Background. Recent studies have shown red blood cell distribution width (RDW) as a marker for severity and prognosis in coronary artery disease patients. Other studies have also correlated RDW with diabetes mellitus and inflammation. However, such correlation and prognosis in patients with concomitant coronary artery disease and diabetes after percutaneous intervention remains unclear.

Material and methods. Our study group comprised of 730 subjects including 700 patients (cases) and 30 normal subjects (control group). Patients who presented with coronary artery disease were divided into diabetic and non-diabetic groups. All patients had RDW measured at admission and percutaneous intervention was done. Follow-up for adverse events was carried out between 6 to 12 months.

Results. RDW was elevated in patients as compared to control group ( $p<0.05$ ). RDW correlated well with inflammatory markers including erythrocyte sedimentation rate, C-reactive protein, $\mathrm{HbA}_{1 \mathrm{c}^{\prime}}$ white blood cells and troponin. RDW was higher with more severe atherosclerosis based on SYNTAX and Gensini scores

Address for correspondence:

Afrasyab Altaf, Assistant Professor

Cardiology Department

Rehman Institute of Medical Sciences, 5/B-2

Hayatabad, Peshawar, Pakistan

Phone: 0092-3075600720

e-mail: afrasyabaltaf@gmail.com

Clinical Diabetology 2020, 9, 3, 174-178

DOI: $10.5603 /$ DK.2020.0017

Received: 21.01.2020
( $p<0.05$ ). Prognosis was found to be worse in patients with high RDW as well as in diabetics.

Conclusions. RDW has positive correlation with other inflammatory marker. It may be used as a marker in determining the severity and prognosis in diabetic patients with coronary artery disease. (Clin Diabetol 2020; 9; 3: 174-178)

Key words: red cell distribution width, coronary artery disease, diabetes

\section{Introduction}

Guidelines for acute ST elevation myocardial infarction (STEMI) patients recommend primary $\mathrm{PCl}$ as the preferred reperfusion strategy [1]. Inflammation plays a critical role in the initiation and propagation of the atherosclerotic process [2, 3]. Numerous inflammatory markers and indices have been studied recently in relation to atherosclerosis. One such marker is red cell distribution width (RDW). Use of RDW has been established in the investigation of the etiology of anemia [4]. Moreover, recent studies are showing an increasing evidence linking elevated RDW with adverse outcomes, in patients with coronary artery disease, heart failure (HF) and with metabolic syndrome. Diabetes mellitus (DM) is an independent risk factor for heart failure (HF) [4-6].

$\mathrm{HbA}_{1 \mathrm{c}}$ is associated with type 2 diabetes patients and is a marker of long term glucose homeostasis as well as adequacy of glycemic control in diabetic patients [7]. $\mathrm{HbA}_{1 \mathrm{c}}$ level increase with age, chronic subclinical 
inflammation and possibly oxidative stress and also in conditions that can adversely affect red blood cell survival. Correlation of RDW and diabetes in coronary artery disease patients has not been studies well.

Therefore, the aim of this exploratory study was to investigate the correlation and prognostic value of RDW in diabetes and coronary artery disease patients.

\section{Patients and methods}

It is a retrospective clinical study which included patients admitted to our hospital with coronary artery disease and diabetes. For the purpose of this analysis patients were divided into low RDW, high RDW, diabetic and non-diabetic groups. ST elevation myocardial infarction (STEMI) was defined as typical chest pain lasting for at least 30 min with new ST-segment elevation at the $J$ point in more than two contiguous precordial or inferior leads (> $0.2 \mathrm{mV}$ in V1 through V3 and > $0.1 \mathrm{mV}$ in the other leads) [8]. Diabetes was defined according to definition previous stated elsewhere [9].

Hypertension was defined as repeated SBP measurements at least $140 \mathrm{~mm} \mathrm{Hg}$, repeated DBP measurements at least $90 \mathrm{~mm} \mathrm{Hg}$ or chronic treatment with antihypertensive medications. Diabetes was recorded when it was reported by the patient and appeared in their medical records or if the patient was receiving regular treatment with oral hypoglycemic agents or insulin. Dyslipidemia was defined as a low-density lipoprotein-cholesterol level above the target according to National Cholesterol Educational Program-3 recommendations, as a high-density lipoprotein-cholesterol level below $40 \mathrm{mg} / \mathrm{dL}$, or chronic treatment with lipidlowering drugs prior to hospitalization [10].

All patients with an ACS received aspirin $(325 \mathrm{mg}$ loading dose) on admission and $100 \mathrm{mg}$ daily as well as clopidogrel (300 mg loading dose for patients $<75$ years of age and $75 \mathrm{mg}$ for patients $>75$ years of age). $\mathrm{PCl}$ was done for all STEMI patients.

Blood samples were taken on admission from all patients at the time of hospitalization and prior to the administration of medication. All hematological measurements were performed using Cobas B221 and 6000 , Roche-Switzerland. Our hospital ethics committee approved this study and informed consent was obtained from all study participants according to the declaration of Helsinki.

\section{Statistical analysis}

Data was analyzed with Statistical Package for the Social Sciences (SPSS 20) for Windows (SPSS Inc., Chicago, Illinois, USA). Continuous data are presented as the mean along with standard deviation. The Kolmogorov-Smirnov test was used to evaluate data normality.
Independent-samples t-test was used to compare two groups showing normal distribution. Categorical variables were summarized as percentages and compared with the chi-square test. Pearson's and Spearman correlation analysis tests were used to determine the correlation between variables. $P$ value less than 0.05 was considered to indicate a significant difference.

\section{Results}

730 subjects were enrolled in this study which included 30 normal and disease free subjects and 700 coronary artery disease patients. Mean RDW in control group was compared to RDW in patients which showed significant difference [(12.4 vs. 14.5) $(p<0.05)]$. All patients underwent percutaneous intervention at about $8 \pm 5$ hours after onset of chest pain. The patients were divided into groups according to RDW and diabetic status. The mean age of the patients was $64 \pm 7.92$ years. Patients were assessed at baseline according to RDW levels (low and high). There was significant difference between low and high RDW groups with regard to hemoglobin, $\mathrm{HbA}_{1 \mathrm{c}^{\prime}}$ erythrocyte sedimentation rate (ESR), C-reactive protein (CRP), white blood cells (WBC) and troponin levels. Other demographic and clinical baseline characteristics between the two groups did not show any significant difference (Table 1).

Angiographic characteristics and scores of the study groups are presented in Table 2. SYNTAX Score ranged from 5 to 47 and with a mean of $23.75 \pm 10.12$ while Gensini Score ranged from 8 to 10 and with a mean of $48.31 \pm 25.47$. SYNTAX and Gensini score were divided into low and high based on mean values. RDW levels in relation to angiographic scoring were assessed in diabetics $\left(\mathrm{HbA}_{1 \mathrm{c}} \geq 7\right)$ and non-diabetics $\left(\mathrm{HbA}_{1 \mathrm{c}}<7\right)$. RDW levels were significantly higher with high SYNTAX score, Gensini scores and multi-vessel disease and as well as in diabetics $(p<0.05)$ (Table 2$)$.

The Pearson and Spearman correlation coefficients were used to assess the correlations between RDW and various clinical and laboratory variables. Significant positive correlation was found between RDW and age, ejection fraction, ESR, $\mathrm{HbA}_{1 c^{\prime}} \mathrm{WBC}, \mathrm{CRP}$ and troponin while there was significant negative correlation with hemoglobin $(p<0.05)$ (Table 3$)$.

Prognosis was assessed on the basis of five indices (angina, myocardial infarction, heart failure, stroke and death) during a period of 6 months to one year after percutaneous intervention. Comparison was made between low and high RDW groups as well as diabetic and nondiabetics. It showed significant increase in myocardial infarction, heart failure and death in both high RDW and diabetic groups. Moreover, frequency of angina was also more in diabetic group $(p<0.05)$ (Table 4). 
Table 1. Baseline characteristics of study groups according to RDW

\begin{tabular}{lcc}
\hline Variable & RDW $<\mathbf{1 6}(\mathbf{n}=\mathbf{3 0 0})$ & RDW $\geq \mathbf{1 6}(\mathbf{n}=\mathbf{4 0 0})$ \\
\hline Age (years) & $63 \pm 7.10$ & $65 \pm 8.75$ \\
Ejection fraction (\%) & $50.62 \pm 8.65$ & $48.78 \pm 8.10$ \\
Hypertension & $99(33 \%)$ & $140(35 \%)$ \\
Smoking & $69(23 \%)$ & $108(27 \%)$ \\
Renal failure & $30(10 \%)$ & $48(12 \%)$ \\
Hyperlipidemia & $63(21 \%)$ & $96(24 \%)$ \\
HbA ${ }_{1 c}$ & $5.5 \pm 0.5$ & $7.5 \pm 1.0^{*}$ \\
Erythrocyte sedimentation rate $[\mathrm{mm} / \mathrm{h}]$ & $18 \pm 5$ & $39 \pm 10^{*}$ \\
C-reactive protein [mg/L] & $0.7 \pm 0.4$ & $1.9 \pm 0.6^{*}$ \\
Troponin (high sensitivity) [ng/l] & $545 \pm 370$ & $2360 \pm 574^{*}$ \\
Hemoglobin [gm/dl] & $15 \pm 1.0$ & $11 \pm 1.5^{*}$ \\
White blood cells $\left[\times 10^{*} / \mathrm{L}\right]$ & $9.50 \pm 2.32$ & $14.48 \pm 2.25^{*}$ \\
Aspirin & $300(100 \%)$ & $400\left(100^{*}\right)$ \\
Clopidogrel & $291(97 \%)$ & $396(99 \%)$ \\
Beta blocker & $285(95 \%)$ & $372(93 \%)$ \\
Renin antagonists & $264(88 \%)$ & $364(91 \%)$ \\
Lipid lowering agents & $240(80 \%)$ & $336(84 \%)$ \\
\hline
\end{tabular}

${ }^{*} \mathrm{p} \leq 0.05$

Table 2. Relationship between RDW and angiographic variables in diabetic $\left(\mathrm{HbA}_{1 \mathrm{c}} \geq 7\right)$ and non-diabetic groups $\left(\mathrm{HbA}_{1 \mathrm{c}}<7\right)$

\begin{tabular}{lcc}
\hline Variable & \multicolumn{2}{c}{ RDW } \\
\cline { 2 - 3 } & $\mathrm{HbA}_{1 \mathrm{c}}<\mathbf{7}$ & $\mathbf{H b A}_{\mathbf{1 c}} \geq \mathbf{7}$ \\
\hline SYNTAX score & $12.85 \pm 1.10$ & $13.45 \pm 1.7$ \\
Low $(<23)$ & $14.24 \pm 1.15$ & $17.85 \pm 1.14^{*}$ \\
Moderate-high (23-32) & \\
Gensini score & & \\
Low $(<47)$ & $12.55 \pm 1.18$ & $12.55 \pm 1.18$ \\
High $(\geq 47)$ & $14.37 \pm 1.21$ & $18.12 \pm 1.11^{*}$ \\
No. of vessels involved & & \\
Single vessel disease & $11.97 \pm 1.25$ & $14.10 \pm 1.36$ \\
Multi-vessel disease & $13.78 \pm 1.75$ & $18.82 \pm 1.61^{*}$ \\
\hline
\end{tabular}

${ }^{*} \mathrm{p} \leq 0.05$
Table 3. Correlation of RDW with clinical and laboratory variables

\begin{tabular}{lc}
\hline Variable & Correlation coefficient (P) \\
\hline Age & $0.487(0.030)$ \\
Systolic blood pressure & $0.037(0.061)$ \\
Diastolic blood pressure & $0.002(0.346)$ \\
Ejection fraction & $0.030(0.048)$ \\
ESR & $0.561(0.001)$ \\
Serum creatinine & $0.258(0.979)$ \\
BMI & $0.003(0.0686)$ \\
Total cholesterol & $0.014(0.725)$ \\
Hemoglobin & $-0.486(0.025)$ \\
HbA ${ }_{1 c}$ & $0.529(0.001)$ \\
WBC $_{\text {CRP }}$ & $0.580(0.001)$ \\
Troponin & $0.349(0.029)$ \\
\hline
\end{tabular}

ESR - erythrocyte sedimentation rate; BMI — body mass indeks; WBC - white blood cells; CRP - C-reactive protein

Table 4. Prognosis in relation to RDW and diabetes after percutaneous intervention

\begin{tabular}{lcccc}
\hline & RDW $<\mathbf{1 6}(\mathbf{n}=\mathbf{3 0 0})$ & $\mathbf{R D W} \geq \mathbf{1 6}(\mathbf{n}=\mathbf{4 0 0})$ & Non-diabetics $(\mathbf{n}=\mathbf{3 8 0})$ & Diabetics $(\mathbf{n}=\mathbf{3 2 0})$ \\
\hline Angina & $18(6 \%)$ & $28(7 \%)$ & $20(5.26 \%)$ & $26(8.12 \%)^{\dagger}$ \\
Myocardial infarction & $4(1.33 \%)$ & $12(3 \%)^{*}$ & $5(1.32 \%)$ & $11(3.44 \%)^{\dagger}$ \\
Heart failure & $3(1 \%)$ & $14(3.50 \%)^{*}$ & $4(1.05 \%)$ & $13(4.06 \%)^{\dagger}$ \\
Stroke & $0(0 \%)$ & $2(0.50 \%)$ & $1(0.26 \%)$ & $1(0.31 \%)$ \\
Death & $3(1 \%)$ & $10(2.50 \%)^{*}$ & $4(1.05 \%)$ & $9(2.81 \%)^{\dagger}$ \\
\hline
\end{tabular}

${ }^{*} \mathrm{p} \leq 0.05$ comparison between RDW $<16$ and RDW $\geq 16$ groups; ${ }^{\dagger} \mathrm{p} \leq 0.05$ comparison between non-diabetic and diabetic groups 


\section{Discussion}

This study showed good correlation of RDW with other inflammatory markers. The underlying pathophysiologic mechanisms for the association between a high RDW and atherosclerosis is unknown, but it is believed that inflammation might play a role with cytokines induced changes in the red cell membrane, leading to an increased RDW [11]. Erythrocyte malformation and hence increased RDW may be caused by oxidative stress, inflammation, and increase in cholesterol levels in erythrocyte membrane. Inflammation or oxidative stress may cause increased RDW by impairing iron metabolism, inhibiting the production of or response to erythropoietin, and shortening red blood cell survival $[12,13]$.

Lippi et al. [14] showed an increase in RDW levels during acute coronary syndrome and its usefulness for the risk stratification. Previous studies have also assessed the relationship between an increased RDW and poor reperfusion results after STEMI, increased risk and morbidity in hospital as well as mortality [15]. A possible explanation for this result is the intense inflammatory response in the setting of STEMI.

In our study, the RDW levels have been shown to be higher in patients with diabetes. A number of risk factors such as age, hypertension, hyperlipidemia, high CRP levels may play a role [12].

Fatemi et al. showed high RDW levels in patients with two or more vessel disease and with early phase of atherosclerosis [16, 17].

Osadnik et al. [18] demonstrated prognostic value of RDW in stable angina pectoris patients undergoing $\mathrm{PCl}$ by showing higher mortality in these patients with a high RDW level.

RDW is a marker of inflammation and has prognostic value in diabetic patients $[19,20]$. The association between DM and RDW has been initially examined by Subharshree [21] showed correlation between BNP and RDW in diabetic patients with HF. Malandrino et al. [22] demonstrated the relationship between RDW and microvascular and macrovascular complications of diabetes mellitus (DM).

RDW may reflect an underlying inflammatory process and inflammation is considered a vital component in the diabetic disease process as well [4, 23], which led to Sherif et al. [24] suggesting that RDW could be used as a marker of inflammation in type 2 DM.

Elevated glucose levels may affect erythrocytes in multiple ways such as changes in erythrocyte membranes which include increased rigidity, changes in osmotic fragility due to changes in $\mathrm{Na}+/ \mathrm{K}+$-ATPase activity and tubulin acetylation and increased aggregation. Other changes include defective oxygen binding of hemoglobin and alterations in cell as well [25-27]. These changes can result in increase in shear stress on the endothelial wall and increased blood viscosity [28].

\section{Conclusion}

RDW has positive correlation with other inflammatory markers. It is an inexpensive and easily accessible marker which can be used in determining the severity and prognosis in diabetic patients with coronary artery disease after percutaneous intervention. More pronounced inflammatory process may be responsible for increased severity and hence poorer prognosis in diabetic patients.

\section{Conflict of interest}

The authors declare to have no conflict of interest.

\section{REFERENCES}

1. 2017 ESC Guidelines for the Management of Acute Myocardial Infarction in Patients Presenting With ST-Segment Elevation: The Task Force for the Management of Acute Myocardial Infarction in Patients Presenting With ST-Segment Elevation of the European Society of Cardiology (ESC). Eur Heart J 2017;Aug 26:[Epub ahead of print].

2. Hansson G. Inflammation, Atherosclerosis, and Coronary Artery Disease. New England Journal of Medicine. 2005; 352(16): 1685-1695, doi: 10.1056/nejmra043430.

3. Baysal E1, Çetin M, Yaylak B. et.al. Roles of the red cell distribution width and neutrophil/lymphocyte ratio in predicting thrombolysis failure in patients with an ST-segment elevation myocardial infarction. Blood Coagul Fibrinolysis. 2015 Apr. ; 26(3): 274-278, doi: 10.1097/MBC.0000000000000227.

4. Xanthopoulos A, Giamouzis G, Melidonis A, et al. Red blood cell distribution width as a prognostic marker in patients with heart failure and diabetes mellitus. Cardiovascular Diabetology. 2017; 16(1), doi: 10.1186/s12933-017-0563-1.

5. Bessman J, Gilmer P, Gardner F. Improved Classification of Anemias by MCV and RDW. American Journal of Clinical Pathology. 1983; 80(3): 322-326, doi: 10.1093/ajcp/80.3.322.

6. Perlstein T, Weuve J, Pfeffer M, et al. Red Blood Cell Distribution Width and Mortality Risk in a Community-Based Prospective Cohort. Archives of Internal Medicine. 2009; 169(6): 588, doi: 10.1001/archinternmed.2009.55.

7. Jaman MS, Rahman MS, Swarna RR, et al. Diabetes and red blood cell parameters. Ann Clin Endocrinol Metabol. 2018; 2: 001-009.

8. Van de, Bax J, Betriu A, et al. Management of acute myocardial infarction in patients presenting with persistent ST-segment elevation: The Task Force on the Management of ST-Segment Elevation Acute Myocardial Infarction of the European Society of Cardiology. Eur Heart J. 2008; 29: 2909-2945.

9. Diagnosis and Classification of Diabetes Mellitus. Diabetes Care. 2011; 35(Supplement 1): S64-S71, doi: 10.2337/dc12-s064.

10. Fedder D, Koro C, L'Italien G. New National Cholesterol Education Program III Guidelines for Primary Prevention Lipid-Lowering Drug Therapy. Circulation. 2002; 105(2): 152-156, doi: 10.1161/ hc0202.101971.

11. Weiss G, Goodnough L. Anemia of Chronic Disease. New England Journal of Medicine. 2005; 352(10): 1011-1023, doi: 10.1056/ nejmra041809.

12. Aysun Erdem, Ufuk Sadik Ceylan, Aycan Esen, et.al. Clinical usefulness of red cell distribution width to angiographic severity and coronary stent thrombosis. Int J Gen Med. 2016; 9: 319-324. 
13. Tziakas D, Chalikias G, Grapsa A, et al. Red blood cell distribution width - a strong prognostic marker in cardiovascular disease - is associated with cholesterol content of erythrocyte membrane. Clinical Hemorheology and Microcirculation. 2012; 51(4): 243-254, doi: 10.3233/ch-2012-1530.

14. Lippi G, Filippozzi L, Montagnana M, et al. Clinical usefulness of measuring red blood cell distribution width on admission in patients with acute coronary syndromes. Clin Chem Lab Med. 2009; 47(3): 353-357.

15. Azab B, Torbey E, Hatoum $H$, et al. Usefulness of red cell distribution width in predicting all-cause long-term mortality after nonST-elevation myocardial infarction. Cardiology. 2011; 119: 72-80.

16. Fatemi O, Paranilam J, Rainow A, et al. Red cell distribution width is a predictor of mortality in patients undergoing percutaneous coronary intervention. Journal of Thrombosis and Thrombolysis. 2012; 35(1): 57-64, doi: 10.1007/s11239-012-0767-x.

17. Wen $Y$. High red cell distrubition width is closely associated with risk of carotid artery atherosclerosis in patients with hypertension. Exp Clin Cardiol. 2010; 15(3): 37-40.

18. Osadnik T, Strzelczyk J, Hawranek M, et al. Red cell distribution width is associated with long-term prognosis in patients with stable coronary artery disease. BMC Cardiovascular Disorders. 2013; 13(1), doi: 10.1186/1471-2261-13-113.

19. Nada A. Red cell distribution width in type 2 diabetic patients. Diabetes, Metabolic Syndrome and Obesity: Targets and Therapy. 2015: 525, doi: $10.2147 /$ dmso.s85318.

20. Engström G, Smith JG, Persson M, et al. Red cell distribution width, haemoglobin $\mathrm{A} 1 \mathrm{c}$ and incidence of diabetes mellitus.
Journal of Internal Medicine. 2014; 276(2): 174-183, doi: 10.1111/joim.12188.

21. Subhashree AR. Red cell distribution width and serum BNP level correlation in diabetic patients with cardiac failure: a cross sectional study. J Clin Diagn Res. 2014; 8(6): FC01-3.

22. Malandrino N, Wu WC, Taveira TH, et al. Association between red blood cell distribution width and macrovascular and microvascular complications in diabetes. Diabetologia. 2012; 55(1): 226-235.

23. Lippi G, Targher G, Montagnana M, et al. Relation between red blood cell distribution width and inflammatory biomarkers in a large cohort of unselected outpatients. Arch Pathol Lab Med. 2009; 133(4): 628-632.

24. Sherif HRN, Radwan M, Hamdy E, et al. Red cell distribution width as a marker of inflammation in type 2 diabetes mellitus. Life Sci J. 2013; 10(3): 1501-1507.

25. Desouky OS. Rheological and electrical behaviour of erythrocytes in patients with diabetes mellitus. Rom J Biophys. 2009; 19(4): 239-250.

26. Soma P, Pretorius E. Interplay between ultrastructural findings and atherothrombotic complications in type 2 diabetes mellitus. Cardiovascular Diabetology. 2015; 14(1), doi: 10.1186/ s12933-015-0261-9.

27. Nigra A, Monesterolo N, Rivelli J, et al. Alterations of hemorheological parameters and tubulin content in erythrocytes from diabetic subjects. The International Journal of Biochemistry \& Cell Biology. 2016; 74: 109-120, doi: 10.1016/j.biocel.2016.02.016.

28. Singh $M$, Shin $S$. Changes in erythrocyte aggregation and deformability in diabetes mellitus: a brief review. Indian J Exp Biol. 2009; 47(1): 7-15. 